\title{
Paper
}

\section{Digital Diorama: Privacy-Preserving and Intelligible Sensing-Based Real-World Content}

\author{
Naoko Nitta (member) ${ }^{\dagger}$, Noboru Babaguchi (member) ${ }^{\dagger}$
}

\begin{abstract}
This paper proposes a sensing-based real-world content called Digital Diorama, which is a three-dimensional miniature model of the dynamically changing real world created from the real-time data continuously published over the Internet by stationary cameras distributed in public spaces. Digital Diorama is designed to preserve both the privacy of the monitored persons and the intelligibility of the content by superimposing the latest background images and human icons each of which represents a monitored person in the three-dimensional model. By using the data continuously published from 10 stationary cameras installed on one floor of a shopping mall, our prototype Digital Diorama browser was able to construct and dynamically update Digital Diorama in approximately $1 \mathrm{fps}$. The results of subjective evaluations indicated that utilizing appropriate human icons can improve the intelligibility of the content while preserving the privacy of the monitored persons.
\end{abstract}

Key words: Sensor data publishing, Distributed smart cameras, Sensing-based real-world content, Three-dimensional visualization, Privacy preservation

\section{Introduction}

In recent years, many sensors such as weather radar, GPS satellites, wireless access points, and cellular phone towers distributed around the world are constantly obtaining the real world data. The data obtained from these sensors is often published over the Internet in real time for public benefits. For example, Weather Underground ${ }^{1)}$ offers the real-time weather data collected from official and personal weather stations. Google $\mathrm{Maps}^{2)}$ offers the real-time traffic congestion maps by collecting the cars' current positions obtained from their drivers' GPS-enabled cell phones so that the drivers can actively avoid congestion up ahead.

Among the sensors, cameras are often installed in many public spaces for security reasons and are mainly used only by authorized persons such as the owners of the cameras and the law enforcement organization members. However, if the owners publish the real-time images or videos captured by these cameras over Internet, it would help ordinary people observe the world directly and obtain various pieces of information. For example, EarthCam ${ }^{3)}$ offers the real-time images of millions of webcams which can take people on virtual tours around the world.

Received December 5, 2014; Revised March 1, 2015; Accepted April 10, 2015

$\dagger$ Graduate School of Engineering, Osaka University (Osaka, Japan)
One of the problems in publishing the images is that, as the number of cameras increases, interpreting their spatial relationships becomes harder for the viewers. To solve this problem, many methods have been proposed to present the real-time images in a three-dimensional model of the monitored space, which is generally prepared beforehand ${ }^{4) \sim 8)}$. In order to present how persons move around the monitored space, persons are automatically detected from the real-time images and their textures are presented in the three-dimensional model at their estimated positions ${ }^{6) \sim 8)}$.

Another problem in publishing the images is that, although presenting the raw images can be informative for viewers, it can also violate the privacy of the monitored persons. Many methods have been proposed for hiding the persons in the images. Their common approach is to detect persons in the images and then hide their visual appearances by using various image filtering techniques such as blurring, pixelization, scrambling, blackout, inpainting, and averaging ${ }^{9) ~ 22)}$. In order to present the persons in the three-dimensional model with preserving their privacy, these image filters can also be applied to the textures of the persons before presenting them in the three-dimensional model.

Now, most of the existing privacy preservation methods for images are designed for surveillance systems, where the personal information sometimes need to be presented to the viewers. For example, the raw textures of persons are presented to authorized persons ${ }^{9)}$, when 
the persons are in unauthorized areas ${ }^{10)}$, or when the persons are in danger ${ }^{11)}$, so that the viewers can observe them directly to obtain their detailed and accurate information. Further, different types of image filters are applied to the raw textures to intentionally disclose only specific personal information according to the relationships between the monitored persons and the viewers ${ }^{12)}$. Although these systems require the raw textures to be stored at least in some situations to control the disclosure of the personal information adaptively to the current situations, the privacy of the monitored persons is considered to be preserved as the surveillance systems are generally not designed for public use and the raw textures are expected to stay within a local area network.

In order to publish the textures of the monitored persons, their privacy needs to be more securely preserved by applying the image filters to the textures in real time, which can be embedded in smart cameras ${ }^{24)}$, before the publication. This solution would make it difficult to adaptively change the image filters according to the viewers, and additionally, still result in unintentional privacy violation. For example, when the person regions are blacked out to show their postures, viewers can also infer their gender from the shapes of the blacked-out person regions ${ }^{20)}$. Further, how much visual appearances can reveal the identities of persons depends on the viewer's prior knowledge about the monitored persons. For example, when only the faces of persons are masked, some viewers such as their family members or friends may be able to identify them by observing their body appearances ${ }^{22)}$. Thus, even when the image filters are perfectly applied before publishing the textures, controlling what to hide or disclose about the persons by using image filtering techniques is not easy. Additionally, the errors in the automatic person detection or image filtering such as image inpainting can accidentally present their visual appearances.

Based on the above observation, this paper proposes Digital Diorama ${ }^{25)}$, which reconstructs the situations captured by stationary cameras installed in public spaces in an intelligible way without unintentionally violating the privacy of the monitored persons. In terms of the privacy preservation, publishing the images themselves should be avoided when they contain persons. Considering that our target is to visualize the real-world so that the ordinary people can browse various areas to quickly grasp the outline of the situations, presenting only the attribute data of the monitored persons such as their positions in the three-dimensional model would be sufficient ${ }^{23)}$. In addition, a few errors in the attribute data would be tolerable.

Thus, we assume that each camera or camera server has the image processing capabilities to constantly detect persons in the captured images and publishes either the background images without persons or the attribute data of the monitored persons which can not be linked to their identities such as their momentary positions, gender, age, and actions. Then, by using the data published from multiple cameras, Digital Diorama is automatically constructed by the following three processes: 1) view control, 2) background image superimposition, and 3) human icon superimposition. 1) presents the published data only within a specific part of the world according to the view and gaze points which are arbitrarily specified by the viewer. As the published data, 2) and 3) respectively superimpose the latest background image captured by each camera at its corresponding position and human icons at the latest positions of the persons in a three-dimensional model of the real world so that the viewers can observe Digital Diorama as if they directly observe the real world where the monitored persons are visually abstracted. Especially, in 3), different types of human icons can be used so that the personal information about the monitored persons other than their momentary positions can be adaptively presented to the viewers in an intelligible way without unnecessarily or erroneously disclosing their appearances.

Note that extensive work has been done by the computer vision community over the years to automatically obtain the attribute data about the persons in the monitored spaces such as their positions, age, gender from the captured images ${ }^{26) \sim 29)}$. In addition, much work has been done for publishing the attribute data about each person obtained by each camera in a way that both the privacy and information usefulness are simultaneously preserved $^{30)}$. Thus, the focus of this paper is how to reconstruct the real world in the three-dimensional model from such data published in a both privacy-preserving and intelligible way.

\section{Digital Diorama}

Fig.1 shows the system architecture of Digital Diorama. We assume stationary cameras installed in public spaces are connected to a sensor server on the local network. Since the real-time data can only be obtained 


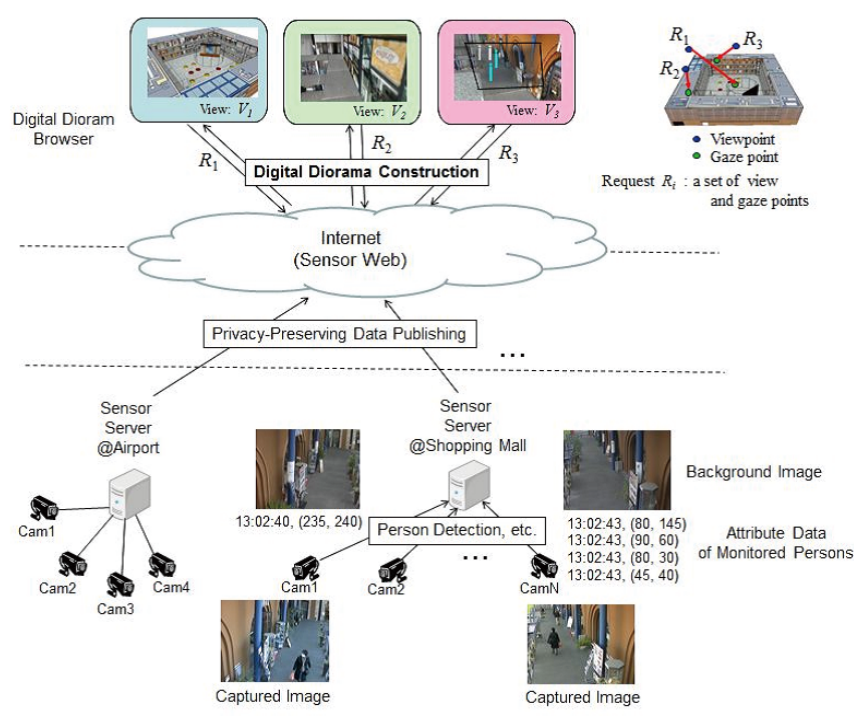

Fig. 1 System architecture.

within the fields of view of the cameras, the sensor server possesses the three-dimensional geometry model of the entire space and their textures as the stationary data of the space. Each camera is calibrated and its position and projection matrix which describes the mapping from the 3-dimensional points in the geometry model to the 2-dimensional points in its image are also stored in the sensor server. The persons in the captured images are constantly detected and their feet positions in the images or in the geometry model are recorded with the time of the detection in the sensor server. When persons are not detected for a set period of time, the mid-frame, which is unlikely to contain any person, can be recorded as a background image. Note that other attribute data of the persons such as the temporal ID, age, gender, and actions which are obtained by other image processing techniques such as person tracking, age/gender estimation, and action recognition can also be recorded with their positions as long as each person can not be confidently linked to an identity in the real world ${ }^{30)}$. When a new data is received from a camera, the sensor server stores it by overwriting the old data. When a request to view a specific space is received from a viewer, the Digital Diorama browser dynamically constructs Digital Diorama by constantly requesting the data published from the sensor server of the space. The data published from the sensor server is summarized in Fig.2.

Fig.3 shows how Digital Diorama is constructed. At any time, the viewer $i$ can give a request $R_{i}$ consisting of the view and gaze points, each of which represents from and around where he/she would like to view the space. When the first request was received from a viewer, Dig-
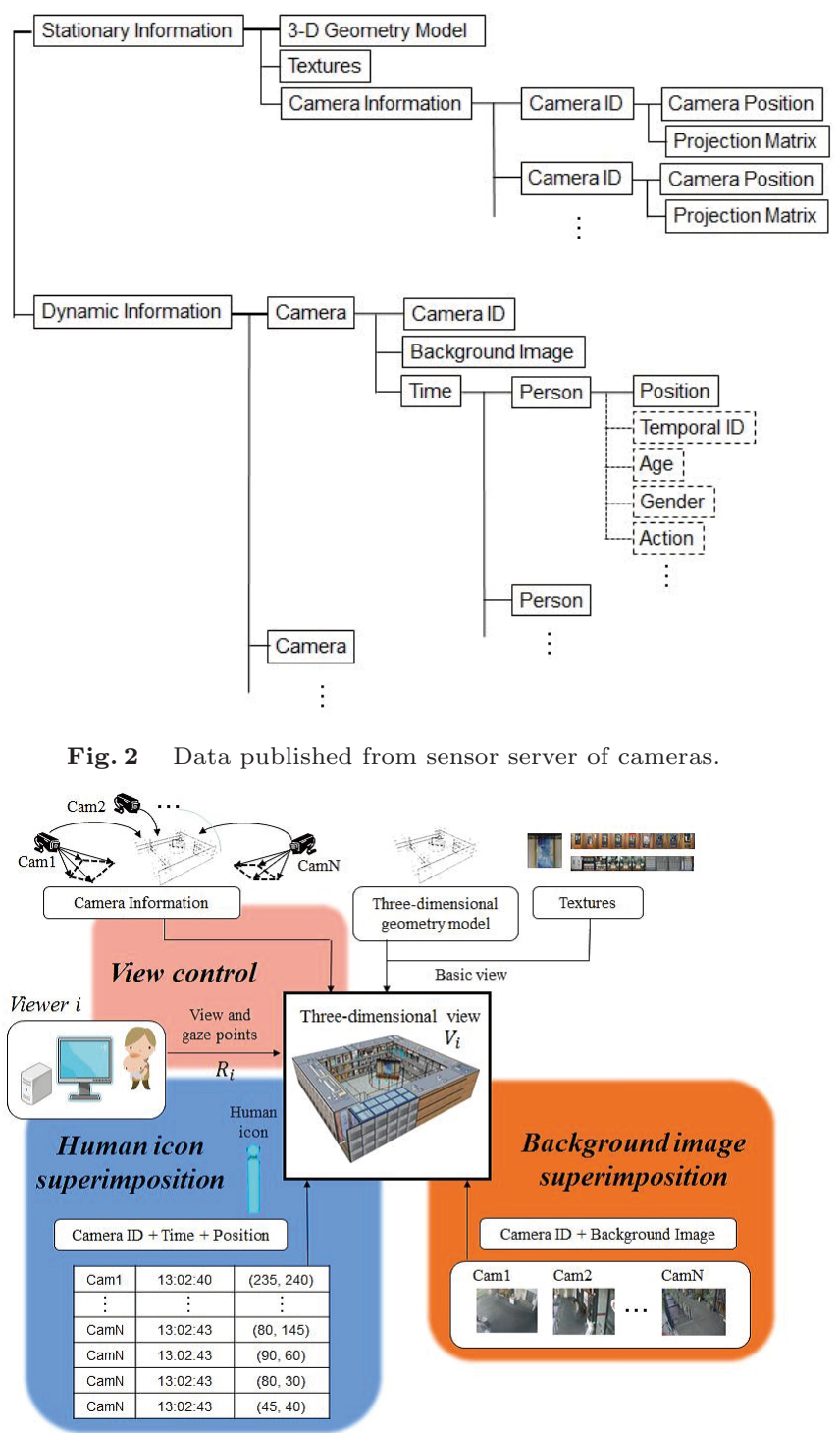

Fig. 3 Digital Diorama construction.

ital Diorama browser firstly obtains the 3-dimensional geometry model and the textures from the sensor server to construct a basic view of the space. Then, with the following 3 processes, Digital Diorama browser repeatedly requests the sensor server for the data which has been published after the last request and superimposes the obtained data in the basic view to construct the view $V_{i}$.

1) View control: Arbitrary areas of interest in the space can be viewed by moving the view and gaze points $R_{i}$ freely. The published data in the corresponding area is presented to the viewer. Fig.1 shows how Digital Diorama presents the distant view of a space $V_{1}$ to a viewer, while it presents a close-up view of a specific area $V_{2}$ to another viewer according to the view and gaze points specified by each viewer.

2) Background image superimposition: In order to present the current situations of the mon- 


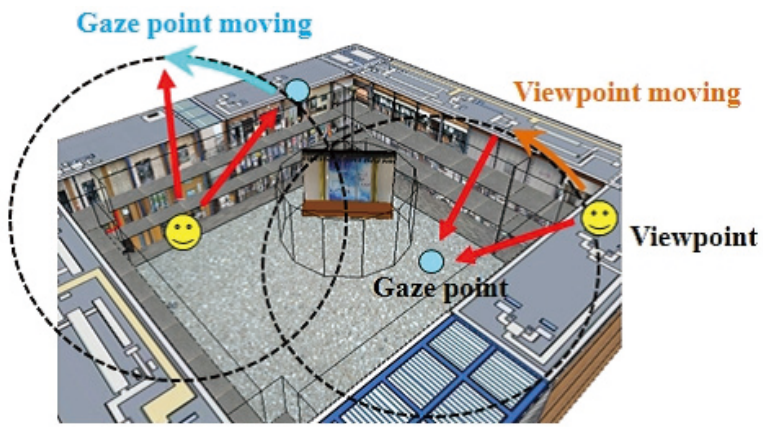

Fig. 4 How to move view and gaze points.

itored space excluding the monitored persons such as the weather conditions, window displays, if each shop is open, etc. the latest background images are superimposed on the basic view so that the viewer can observe an image seamlessly with the basic view when the viewpoint is exactly at its camera position. Fig.1 shows how a background image is presented in the view $V_{3}$ when the corresponding camera position is specified as the viewpoint.

3) Human icon superimposition: Human icons are superimposed at the latest positions of the detected monitored persons in the basic view. Fig.1 shows how each person is represented by an anonymous human icon in the view $V_{3}$.

The details of each process are described in the following subsections.

\subsection{View control}

The viewer can specify arbitrary view and gaze points. The viewpoint corresponds to the position of viewer's eyes in the three-dimensional model and the gaze point corresponds to the position over the line of sight. Changing the view and gaze points enables the viewer to view the areas of interest freely. As shown in Fig.4, the viewpoint can be moved on the surface of the sphere whose center is the current gaze point and radius equals the distance between the view and gaze points. Similarly, the gaze point can be moved on the surface of the sphere whose center is the current viewpoint and radius equals the distance between the view and gaze points. Additionally, the viewpoint can also be moved forward or backward along the line determined by the view and gaze points. Then, the published data in the corresponding view is presented to the viewer. The details of how the published data is presented are described in the following subsections.

\subsection{Image superimposition}

Presenting the images captured by cameras in the basic view enables the viewers to observe the current

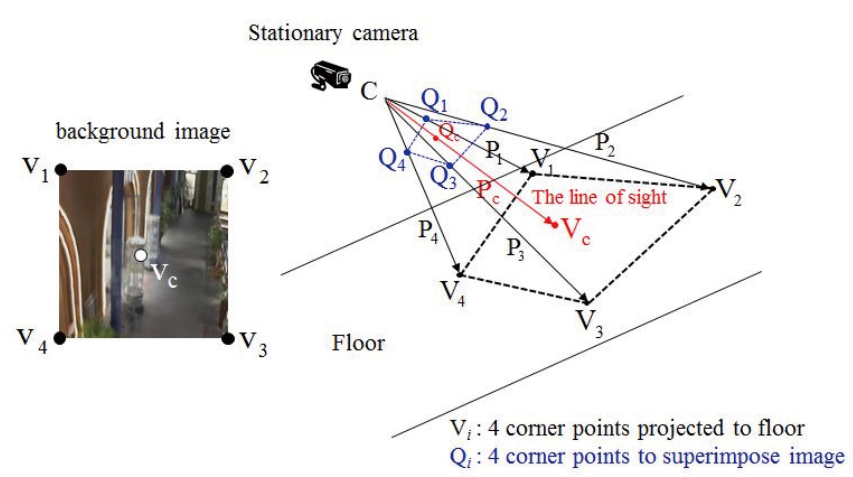

Fig. 5 How to superimpose an image in the threedimensional model .

situations of the monitored space within the confined space of the cameras' fields of view. However, since the visual appearances of persons captured by cameras are sensitive to their privacy, the images to be presented should not contain persons. Although the image inpainting techniques can be used to remove the persons from the captured images, the errors in image inpainting techniques can lead to unintended privacy violation. Since the situations which can be observed in the images would not change so frequently, one way to publish images would be to use the raw images only when no person is detected. In this case, since the errors in the person detection can also result in the privacy violation, the mid-frame should be published only when no person is detected for a certain period of time.

As illustrated in Fig.5, the published image can be superimposed by arranging the image in the threedimensional model so that each corner point of the superimposed image $\mathbf{v}_{i}(i=1,2,3,4)$ is on the line determined by their corresponding points in the threedimensional model $\mathbf{V}_{i}(i=1,2,3,4)$ and the given camera position $\mathbf{C}$, and is on the plane vertical to the line of the sight of the camera.

$\mathbf{V}_{i}$ can be calculated as follows. Let $H$ denote the two-dimensional projective matrix, which represents the relationship between the image coordinates and the floor coordinates in the three-dimensional model. $\mathbf{V}_{i}=$ $\left(X_{i}, Y_{i}, Z_{i}\right)$ is determined as $X_{i}=X_{i}^{\prime} / Z_{i}^{\prime}, Y_{i}=Y_{i}^{\prime} / Z_{i}^{\prime}$, where $\mathbf{V}_{i}^{\prime}=\left(X_{i}^{\prime}, Y_{i}^{\prime}, Z_{i}^{\prime}\right)$ is obtained as $\mathbf{V}_{i}^{\prime}=H \mathbf{v}_{i}$. $Z_{i}$ is the height of the floor and is determined from the threedimensional geometry model of the monitored space. $\mathbf{V}_{\mathrm{c}}$, the point in the three-dimensional model corresponding to the image center point $\mathbf{v}_{\mathrm{c}}$, is calculated likewise. The line of sight of the camera is represented by the line determined by $\mathbf{C}$ and $\mathbf{V}_{\mathrm{c}}$. An arbitrary vector $\mathbf{x}$, which is vertical to the line, satisfies $\mathbf{x} \cdot \mathbf{P}_{\mathrm{c}}=0$, 


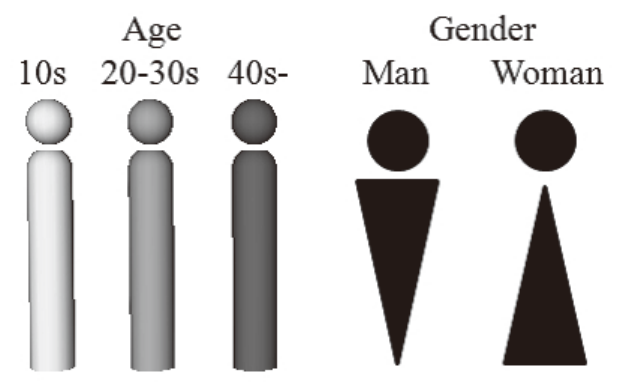

Fig. 6 Examples of human icons presenting the attribute data of the monitored persons.

where $\mathbf{P}_{\mathrm{c}}=\mathbf{V}_{\mathrm{c}}-\mathbf{C}$. Since each corner point of the superimposed image is on the line determined by $\mathbf{C}$ and $\mathbf{V}_{i}$ and is on the plane vertical to the line of sight of the camera, the following equation is obtained.

$$
\left(t_{i} \mathbf{P}_{i}-\alpha \mathbf{P}_{\mathrm{c}}\right) \cdot \mathbf{P}_{\mathrm{c}}=0,
$$

where $\mathbf{P}_{i}=\mathbf{V}_{i}-\mathbf{C}$ and $\alpha$ is a constant which determines the distance between the camera position and the superimposed image. By solving this equation with respect to $t_{i}$, the corner points in the three-dimensional model, $\mathbf{Q}_{i}$, on which the corner points of the image $\mathbf{v}_{i}$ are superimposed, are obtained as

$$
\mathbf{Q}_{i}=\frac{\alpha\left|\mathbf{P}_{\mathrm{c}}\right|^{2}}{\mathbf{P}_{i} \cdot \mathbf{P}_{\mathrm{c}}} \mathbf{P}_{i}+\mathbf{C} .
$$

The background image is presented in the quadrilateral consisting of $\mathbf{Q}_{i}(i=1,2,3,4)$.

When the viewer chooses a camera in Digital Diorama, the viewpoint can be moved automatically to the position of the camera since the superimposed image can be presented seamlessly only when the viewpoint is exactly at the same position as the camera position. To show the viewers the position and field of the view of each camera, Digital Diorama can present its view frustum, which is determined by the 4 points $\mathbf{V}_{i}$ $(i=1,2,3,4)$ and the camera position C. Presenting the view frustums enables the viewers to see where in the Digital Diorama the real-time data can be observed.

\subsection{Human icon superimposition}

Superimposing human icons at the latest positions of the detected persons in the basic view enables the viewers to observe the current congestion situation in the monitored space without violating the privacy of persons in the space. In the area where the fields of view of more than two cameras overlap, the latest published data is prioritized.

If each camera or the sensor server is embedded with the person detection techniques ${ }^{26)}$, the momentary positions of the persons can be published. Additional attribute data about the persons can be published by

\begin{tabular}{ll}
\multicolumn{1}{c}{ Table $1 \quad \begin{array}{c}\text { Specification of Digital Diorama browsing } \\
\text { client. }\end{array}$} \\
\hline OS & Windows XP Professional Service Pack3 \\
CPU & Intel Xeon $3.73 \mathrm{GHz}$ \\
RAM & 3.00 GBytes \\
GPU & ATI FireGL v3400 \\
Display Size & $1280 \times 1024$ \\
Graphics API & OpenGL 2.0
\end{tabular}

other image processing techniques ${ }^{26)}{ }^{29}$. For example, the temporal ID of the persons, which are only valid in the field of view of each camera or in the monitored space, can be obtained by person tracking or person recognition in a single- or multi-camera environment. When the temporal IDs are available, the human icons can be presented with their trajectories. Age or gender of the persons, which are visually observable demographic data, can also be obtained by age/gender estimation. Different human icons can be used according to the published demographic data. For example, as shown in Fig.6, human icons of different colors and shapes can present their age and gender. Such presentation can provide more information than the congestion situation such as the customer segment of each shop in a shopping mall.

\section{Experiments}

We have implemented a prototype system for constructing Digital Diorama for a shopping mall located in Kyoto, Japan, where 10 stationary cameras connected to a sensor server are installed on one floor (about 314 square meters).

We set up a PC with Digital Diorama browser with a large-screen TV in an area in the shopping mall where the customers can visit and observe Digital Diorama to compare the presented view and the actual situation in the shopping mall. Table 1 shows the specification of the PC on which Digital Diorama browser is implemented. For the view control, we use SONY DUALSHOCK 2 game pad with two analog sticks, each of which is used to move the view and the gaze points respectively, so that viewers can easily and intuitively move the view and gaze points. Fig.7 shows the key bindings for the game pad.

Fig. 8 shows the example views constructed by the 3 processes: 1) view control, 2) background image superimposition, and 3) human icon superimposition. The view can be controlled using the game pad from a distant view to a close-up view of a person as shown in Fig.8 (a) and (b). In addition, in a basic view presented when the viewpoint is the position of a camera as 


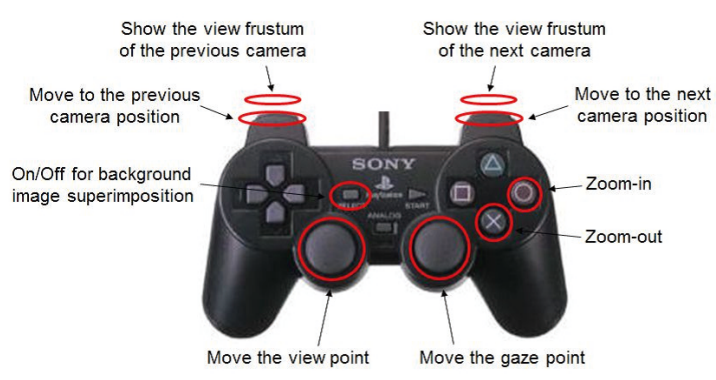

Fig. 7 View control by game pad.

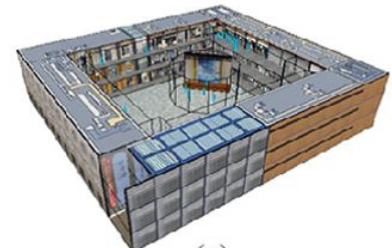

(a)

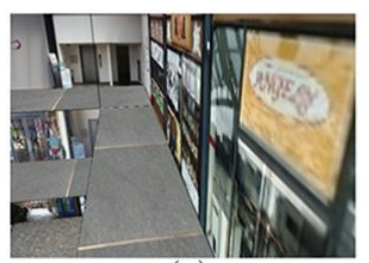

(c)

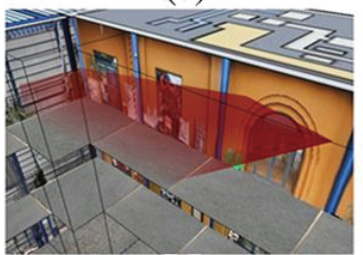

(e)

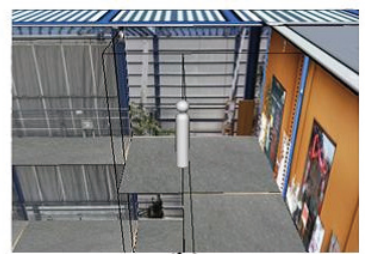

(b)

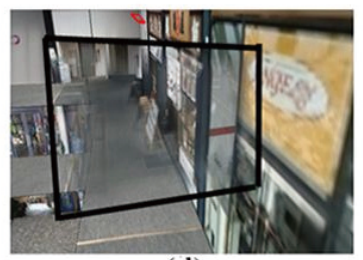

(d)

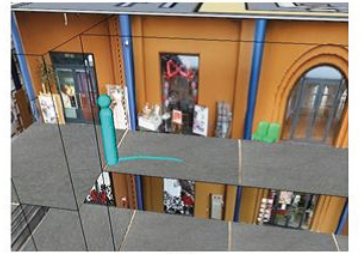

(f)
Fig. 8 Example views of Digital Diorama. (a), (b) View control. (c) - (e) Background image superimposition. (f) Human icon superimposition with trajectory

shown in Fig.8 (c), the background image captured by the camera is superimposed and seamlessly presented as shown in Fig.8 (d). Here, the image is highlighted by a heavy line. The view frustum can be presented as shown in Fig.8 (e). When the temporal ID of the monitored person is published as the result of the person tracking from the sensor server, the trajectories of the persons can be presented as shown in Fig.8 (f).

Table 2 shows the framerate of the construction of Digital Diorama when requesting no real-time data, only the attribute data of the monitored persons, and both the attribute data of the monitored persons and the background images published from all cameras. It can be seen that, the three-dimensional view of Digital Diorama with the human icons and background images published from 10 cameras is constructed in about 1 frame per second. Since the amount of data which needs to be transferred differs largely between the images and the attribute data, the view only with the hu-
Table 2 Processing time in a shopping mall.

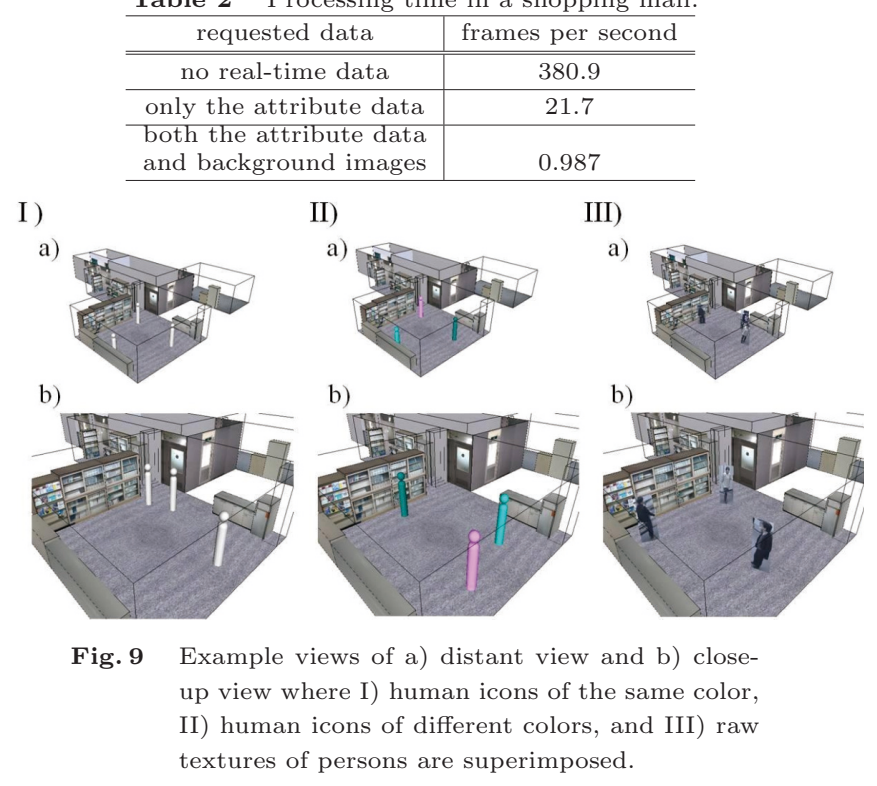

man icons can be constructed in more than 20 frames per second. Let us note that there exist a lot of techniques for person detection and other image processing techniques for extracting the attribute data of the monitored persons ${ }^{26)}{ }^{29)}$ and their processing time depends on the techniques to be used. Since the amount of the attribute data of the monitored person is small, once the attribute data is stored in the camera server, the effect of the number of persons in the monitored space to the framerate of Digital Diorama is negligible.

The attribute data of the monitored persons other than their momentary positions can be presented by changing the human icons. The following subsections introduce two examples of using different human icons with their subjective evaluations.

\section{1 Attribute-based Human Icon Superim- position}

In order to examine how different types of human icons affect the intelligibility and the privacy concerns of Digital Diorama, we prepared three types of human icons: I) human icons of the same color, II) human icons of different colors depending on the age and gender of the persons, and III) raw textures of the persons. For II), blue is used to represent men while pink is used to represent women, and elder persons are represented by deeper colors. I) and II), both of which present human icons according to their attribute information, are expected to be more appropriate in terms of preserving their privacy than III), which requires their raw textures to be published and presented. On the other hand, III) is expected to achieve the highest intelligibility by providing all the visually observable information about the persons, while I) provides only the positions of persons 


\section{$\square 1 \square 2 \square 3 \square 4 \square 5$}

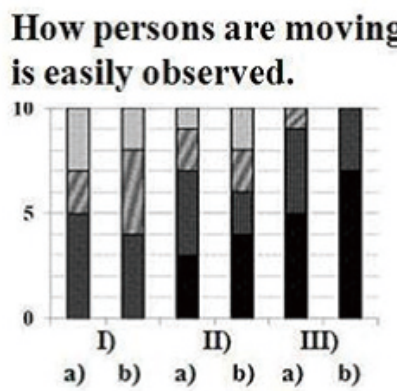

The age of persons is easily observed.

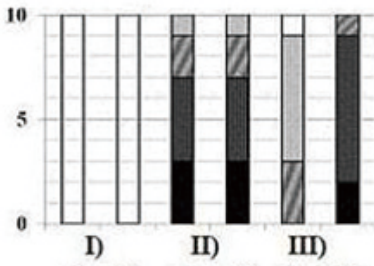

a) b) a) b) a) b)
The gender of persons is easily observed.

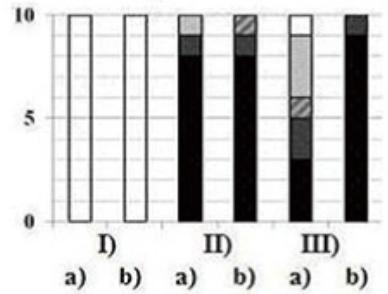

Fig. 10 Results of questionnaire for attribute-based human icon superimposition.

and II) additionally provides their age and gender.

The three types of human icons are superimposed in the basic view and presented to the viewer as two different types of view a) distant view, where the viewer can look over the monitored space and b) close-up view, where the viewer can look at a specific area in the space. Let us note here that, due to the difficulty in obtaining the permission from the shopping mall to present the visual appearances or the attribute data of the customers, we constructed Digital Diorama for our laboratory for this experiment. Fig.9 shows the example views. All the views were constructed when two men in their $20 \mathrm{~s}$ and a woman in her 20s are walking around a room in our laboratory.

We conducted a questionnaire composed of 4 questions. We divided 30 men and women from their 20 s to 40s into 3 disjoint groups and each group watched the views a) and b) with one type of human icons, and answered questions using the scores of 5: Strongly agree, 4: Agree, 3: Neither, 2: Disagree, or 1: Strongly disagree. Fig.10 shows each question and the result. The results indicate that how persons move around is easily observed by a majority of viewers for any type of human icons, but the best result was obtained for III) raw textures, since the moving directions of the persons are not presented by the human icons. When the persons are represented by human icons, the viewers sometimes can not distinguish each person when they

cross each other or move around in a small area. The gender or age of the persons is easily observed for II) human icons of different colors both in a) distant and b) close-up views, while the results differ largely between a) and b) for III) raw textures. While more than half viewers were satisfied with the privacy preservation for I) and II), much fewer viewers were satisfied for III), especially for b) close-up view.

In summary, human icons can clearly present only the personal information which is intentionally disclosed both in the distant and close-up views with satisfactorily preserving their privacy. The raw textures provide the easiest understanding of how persons move around. However, the personal information such as their age and gender can be observed easily only in the close-up view, where the monitored persons feel their privacy violated. These results show that using appropriate human icons according to the attribute data of the monitored persons as in II) can increase the intelligibility of the content without violating the privacy of the monitored persons.

\section{2 Viewer-based Human Icon Superimposi- tion}

The previous subsection introduces a case where the human icons are changed only according to the demographic data of the monitored persons which is published from cameras in a privacy-preserving way, so that each monitored person is presented in the same way to all viewers. Additionally, the same monitored person can be presented differently to different viewers by disclosing different attribute data according to the viewer. For example, by authenticating the viewers, more attribute data can be disclosed to authorized persons, while only the positions can be disclosed to unauthorized persons. Further, even in public spaces, the monitored persons can be recognized or identified if their identity-related data such as faces and gaits are registered beforehand with their agreement so that their positions can be published with their temporal IDs. A group of persons such as family or friends can register their temporal IDs in their Digital Diorama browser so that they can observe where the group members are in Digital Diorama. In order to evaluate how ordinary people feel about the intelligibility and privacy of the monitored persons in Digital Diorama, we implemented such viewer-based human icon superimposition, where the human icons are changed according to the relationships of the monitored persons and viewers.

Although there are many visual person identification 


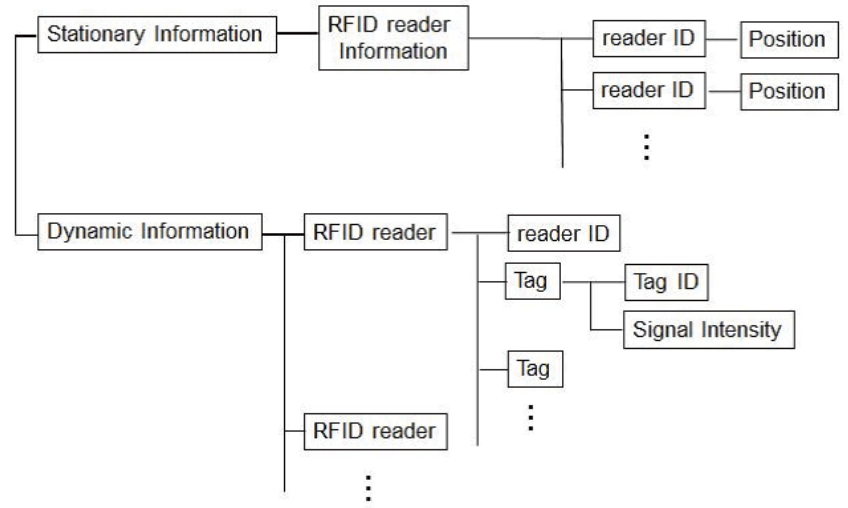

Fig. 11 Data published from sensor server of RFID readers.

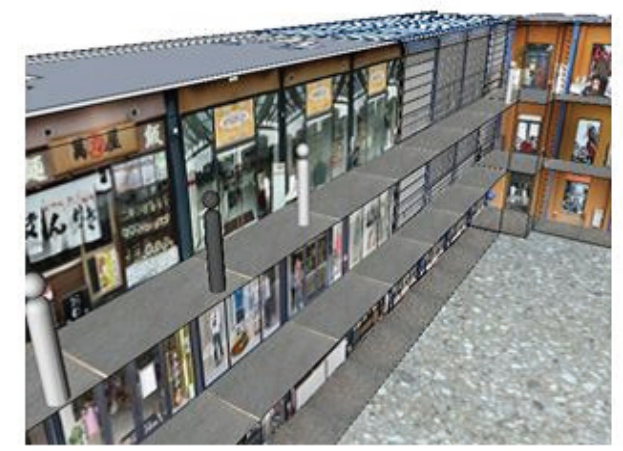

Fig. 12 Viewer-based human icon superimposition.

methods based on faces or gaits, due to the appearance changes of the same persons or occlusion, using Wi-Fi, GPS, or RFID is a more practical solution for person identification. Further, in order to show that the data published from other types of sensors can be integrated in the same system architecture, we additionally installed 11 RFID readers in the shopping mall, which are connected to another sensor server. We distributed RFID tags to the family or friends who signed up at the entrance of the shopping mall and registered the list of the tag IDs as a group to the Digital Diorama browser.

Fig.11 shows the data published from the sensor server of RFID readers. The signal intensities of RFID tags captured by each RFID reader imply their distances from the reader. The rough position of each RFID tag can be inferred from its distances from multiple RFID readers. Thus, when a request to view a specific space is received from a viewer, the Digital Diorama browser simultaneously requests the data published from the sensor servers of the RFID readers and the cameras. By matching the rough positions inferred from signal intensities of RFID tags to the positions of the monitored persons obtained from cameras ${ }^{31)}$, each tag ID can be assigned to at most one of the positions of the monitored persons.
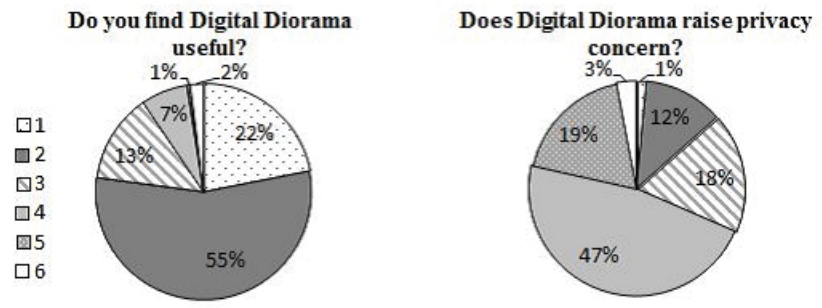

Fig. 13 Results of questionnaire composed of 2 questions for viewer-based human icon superimposition .

We also installed an RFID reader in the area where the PC with Digital Diorama browser is set up, with which the viewer carrying the RFID tag distributed at the entrance can be identified. The persons who are registered with the viewer as a group are represented by black human icons, while other persons are represented by white human icons as shown in Fig.12. In this way, the same monitored person can be presented differently according to the viewer.

We offered the system demonstrations to the customers at the shopping mall three times every couple of months and conducted questionnaire surveys composed of 2 questions and obtained responses from 200 male and female customers in their teens to over sixties in total using the scores of 1: Strongly agree, 2: Agree, 3: Neither, 4: Disagree, 5: Strongly disagree, or 6: Don't know. Note that the published data can contain errors and the customers answered questionnaire by observing the actual situations in the shopping mall and Digital Diorama at the time. Fig.13 shows each question and the result. From the results, nearly $80 \%$ of customers found Digital Diorama useful even with some errors in the attribute data, while only $13 \%$ are concerned about the privacy issue.

On the last day, we added 7 other more detailed questions about the usability of Digital Diorama and obtained responses from 40 customers. Fig.14 shows each question and the result. $87 \%$ of customers responded positively to the reality of the appearances of the threedimensional model of the shopping mall created from the textures pre-mapped to the basic view. For the view control, $62 \%$ of customers responded positively to the ease of view control using a gamepad. In contrast, less than half customers agreed that the background images are presented seamlessly. Improving how to superimpose the background images especially when the view point does not correspond to the camera position would be our future work. As for the human icons, a majority of customers responded positively to all 4 questions. While a majority (74\%) of customers felt that the pri- 


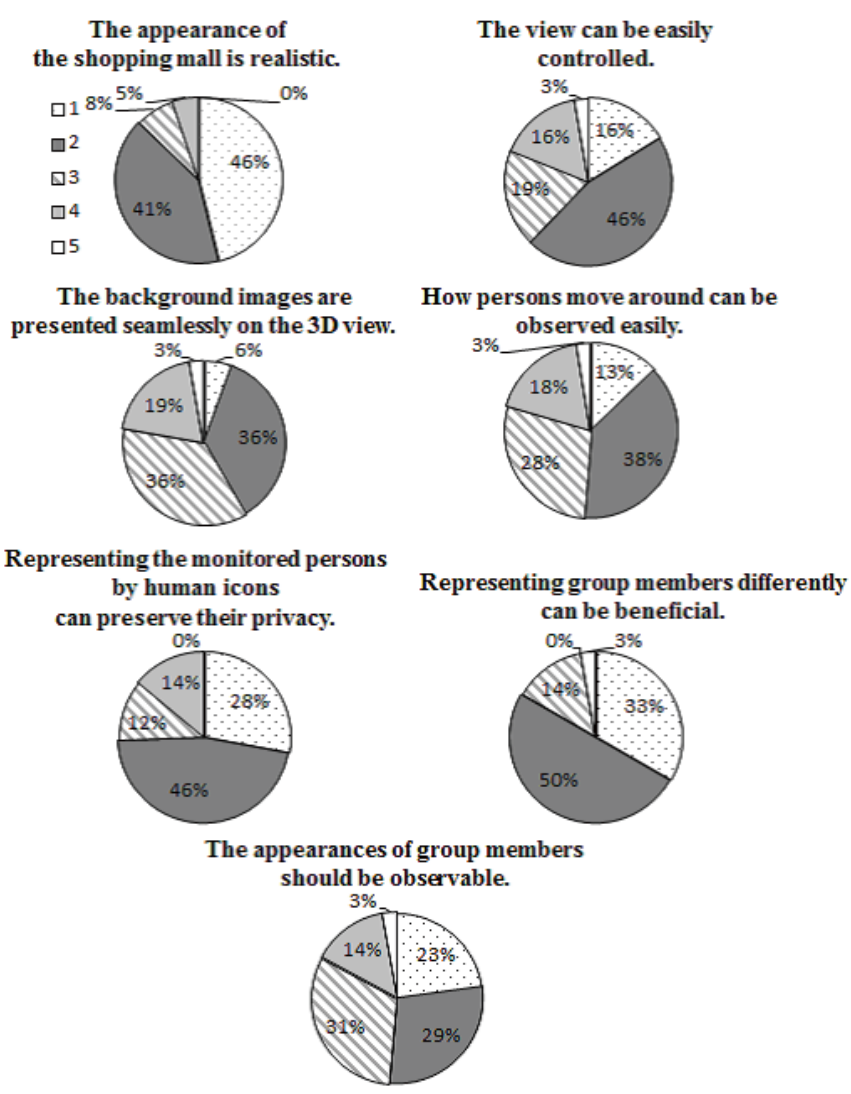

Fig. 14 Results of questionnaire composed of 7 questions for viewer-based human icon superimposition.

vacy of the monitored persons were preserved, fewer customers (51\%) felt that how they moved around were easily observable. As discussed in Section 3.1, publishing the moving directions of the persons as the default attribute data with their positions would improve the intelligibility. Further, a majority (83\%) of customers agreed that presenting the persons who are in the same group with the viewer differently can be beneficial, for example, to find their children when they get lost. On the other hand, many customers (52\%) felt that their visual appearances should be observable since only their positions are observable in the demonstrations. This result also infers the importance of using different types of human icons according to the attribute data of the monitored persons as described in Section 3.1.

\section{Conclusion}

This paper proposed a sensing-based real-world content, Digital Diorama, which presents the real-time data published from multiple stationary cameras capturing the public places. By considering both the intelligibility of the content and the preservation of the privacy of the monitored persons, Digital Diorama is constructed only from the the background images and the attribute data of the persons in the monitored space. Digital Diorama can be updated in about 1 frame per second by dynamically obtaining the data published from 10 stationary cameras installed in a shopping mall. In addition to the results of the survey questionnaires, this confirms the feasibility of Digital Diorama in real environments. The results of evaluation for human icons indicated that utilizing an appropriate human icon based on the attribute data of each monitored person would make the content more intelligible, sometimes even compared to when the raw textures are presented, without violating their privacy. Our main future work is to improve how to present the background images. This work was partly supported by JSPS Grant-in-Aid for Scientific Research.

\section{References}

1) Weather Underground, http://www. wunderground.com/

2) Google Maps, http://maps.google.com

3) EarthCam, http://www.earthcam.com/

4) H. S. Sawhney, A. Arpa, R. Kumar, S. Samarasekera, M. Aggarwal, S. Hsu, D. Nister, and K. Hanna: "Video Flashlights - Real Time Rendering of Multiple Videos for Immersive Model Visualization," Eurographics Workshop on Rendering, pp. 157-168 (2002)

5) G. de Haan, J. Scheuer, R. de Vries, and F. H. Post: "Egocentric Navigation for Video Surveillance in 3D Virtual Environments," IEEE Symp. on 3D User Interfaces, pp. 103-110 (2009)

6) I. O. Sebe, J. Hu, S. You, and U. Neumann: "3D Video Surveillance with Augmented Virtual Environments," ACM SIGMM Int'l Workshop on Video Surveillance, pp.107-112 (2003)

7) S. Fleck, F. Busch, P. Biber, and W. Strasser: "3D Surveillance - A Distributed Network of Smart Cameras for Real-Time Tracking and its Visualization in 3D," Computer Vision and Pattern Recognition Workshop, (2006)

8) A. Girgensohn, D. Kimber, J. Vaughan, T. Yang, F. Shipman, T. Turner, E. Rieffel, l. Wilcox, F. Chen, and T. Dunnigan: "DOTS: Support for Effective Video Surveillance," ACM Int'l Conf. on Multimedia, pp. 423-432 (2007)

9) A. J. Aved and K. A. Hua: "A General Framework for Managing and Processing Live Video Data with Privacy Protection," Multimedia Syst., 18, 2, pp.123-143 (2012)

10) J. Wickramasuriya, M. Datt, S. Mehrotra, and N. Venkatasubramanian: "Privacy Protecting Data Collection in Media Spaces," ACM Int'l Conf. on Multimedia, pp.48-55 (2004)

11) S. Moncrieff, S. Venkatesh, and G. West: "Dynamic Privacy Assessment in a Smart House Environment Using Multimodal Sensing," ACM Trans. Multimedia Comput., Commun. \& Appl., 5, 2, 10 (2008)

12) K. Chinomi, N. Nitta, Y. Ito, and N. Babaguchi: "Prisurv: Privacy Protected Video Surveillance System Using Adaptive Visual Abstraction," Int'l Conf. on Advances in Multimedia Modeling, pp.144-154 (2008)

13) F. Z. Qureshi, "Object-Video Streams for Preserving Privacy in Video Surveillance," Int'l Conf. on Advanced Video and Signal Based Surveillance, pp.442-447 (2009)

14) S. Fleck and W. Strasser: "Privacy Sensitive Surveillance for Assisted Living - A Smart Camera Approach, "Handbook of Ambient Intelligence and Smart Environments, Springer US, pp.9851014 (2010)

15) E. Newton, L. Sweeney, and B. Malin: "Preserving Privacy by De-identifying Facial Images," IEEE Trans. Knowledge \& Data Eng., 17, 2, pp.232-243, (2005)

16) F. Dufaux and T. Ebrahimi: "Scrambling for Privacy Protection in Video Surveillance Systems," IEEE Trans. Circuits \& Syst. for Video Technology, 18, 8, pp.1168-1174 (2008)

17) S. Tansuriyavong and S. Hanaki: "Privacy Protection by Concealing Persons in Circumstantial Video Image," Workshop on Perceptive User Interfaces, pp.1-4 (2001)

18) M. V. Venkatesh, S.-C. S. Cheung, and J. Zhao: "Efficient Object-Based Video Inpainting," Pattern Recognition Letters, 
30, 2, pp.168-179 (2009)

19) P. Korshunov, A. Melle, J.-L. Dugelay, and T. Ebrahimi: "Framework for Objective Evaluation of Privacy Filters," SPIE vol.8856, Applications of Digital Image Processing XXXVI, (2013)

20) P. Korshunov, C. Araimo, F. D. Simone, C. Velardo, J.-L. Dugelay, and T. Ebrahimi: "Subjective Study of Privacy Filters in Video Surveillance," IEEE Int'l Workshop on Multimedia Signal Processing, pp.378-382 (2012)

21) J. Schiff, M. Meingast, D. K. Mulligan, S. Sastry, and K. Y. Goldberg: "Respectful Cameras: Detecting Visual Markers in Real-Time to Address Privacy Concerns," Protecting Privacy in Video Surveillance, Springer London, pp.65-89 (2009)

22) D. Chen, Y. Chang, R. Yan, and J. Yang: "Tools for Protecting the Privacy of Specific Individuals in Video," EURASIP J. Advances in Signal Process. 2007 (2007)

23) RaFlow, http://ralc.cec-ltd.co.jp/lineup/raflow.html

24) A. Senior, S. Pankanti, A. Hampapur, L. Brown, Y.-L. Tian, A. Ekin, J. Connell, C. F. Shu, and M. Lu: "Enabling Video Privacy Through Computer Vision," IEEE Security \& Privacy, 3, 3, pp.50-57 (2005)

25) T. Takehara, N. Nitta, and N. Babaguchi: "Three-level Privacy Control for Sensing-Based Real-World Content Digital Diorama," ACM Int'l Conf. on Internet Multimedia Computing and Service, pp.17-20 (2011)

26) M. Enzweiler and D. M. Gavrila: "Monocular Pedestrian Detection: Survey and Experiments," IEEE Trans. Pattern Analysis and Machine Intelligence, 31, 12, pp.2179-2195 (2009)

27) J. K. Aggarwal and M. S. Ryoo: "Human Activity Analysis: A Review," ACM Computing Surveys, 43, 3, 16 (2011)

28) C. B. Ng, Y. H. Tay, B.-M. Goi: "Recognizing Human Gender in Computer Vision: A Survey," PRICAI 2012: Trends in Artificial Intelligence, Springer Berlin Heidelberg, pp.335-346 (2012)

29) Y. Fu, G. Guo, and T. S. Huang: "Age Synthesis and Estimation via Faces: A Survey," IEEE Trans. on Pattern Analysis and Machine Intelligence, 32, 11, pp.1955-1976 (2010)

30) B. C. M. Fung, K. Wang, R. Chen, and P. S. Yu: "PrivacyPreserving Data Publishing: A Survey on Recent Development," ACM Computing Surveys, 42, 4, 14 (2010)

31) D. Nakashima, Y. Ito, and N. Babaguchi: "Human Identification in Surveillance Video Based on Tracking via Camera Footage and ID Updating via RFID Systems," Int'l Workshop on Computer Vision and Its Application to Image Media Processing, pp.58-62 (2009)

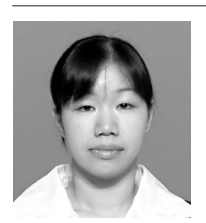

Naoko Nitta Naoko Nitta received the B.E., M.E. and Ph.D. degrees in Engineering Science from Osaka University, in 1998, 2000 and 2003, respectively. She is currently a Lecturer in Graduate School of Engineering, Osaka University. From 2002 to 2004, she was a research fellow of the Japan Society for the Promotion of Science. From 2003 to 2004, she was a Visiting Scholar at Columbia University, New York. Her research interests are in the areas of video content analysis and image/audio processing. She received Best Paper Award of 2006 Pacific-Rim Conference on Multimedia (PCM2006). She is a member of IEEE, ACM, IEICE, and ITE.

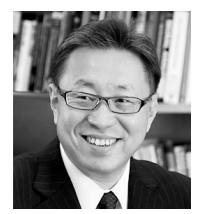

Noboru Babaguchi Noboru Babaguchi received the $\mathrm{B} . \mathrm{E}$, $\mathrm{M} \mathrm{E}$ and $\mathrm{Ph} \mathrm{D}$, degrees in communication engineering from Osaka University, in 1979, 1981 and 1984, respectively. $\mathrm{He}$ is currently a Professor of the Department of Information and Communications Technology, Osaka University. From 1996 to 1997, he was a Visiting Scholar at the University of California, San Diego. His research interest include image analysis, multimedia computing, and intelligent systems. He received Best Paper Award of 2006 Pacific-Rim Conference on Multimedia, and Fifth International Conference on Information Assurance and Security. $\mathrm{He}$ is on the editorial board of Multimedia Tools and Applications, Advances in Multimedia, and New Generation Computing. He served as a General Co-chair of the 14th International MultiMedia Modeling Conference, ACM Multimedia 2012, and so on. He is a Fellow of IEICE, a Senior Member of IEEE, and a member of ACM, IPSJ, ITE and JSAI. 